\title{
Odd order flag-transitive affine planes of dimension three over their kernel
}

\author{
Ronald D. Baker, C. Culbert*, Gary L. Ebert* and Keith E. Mellinger*
}

Dedicated to Adriano Barloti on the occasion of his 80 th birthday

\begin{abstract}
With the exception of Hering's plane of order 27, all known odd order flag-transitive affine planes are one of two types: admitting a cyclic transitive action on the line at infinity, or admitting a transitive action on the line at infinity with two equal-sized cyclic orbits. In this paper we show that when the dimension over the kernel for these planes is three, then the known examples are the only possibilities for either of these two types. Moreover, subject to a relatively mild gcd condition, one of these two actions must occur. Hence, subject to this gcd condition, all odd order three-dimensional flag-transitive affine planes have been classified.
\end{abstract}

\section{Introduction}

Let $q=p^{e}$ be an odd prime power. In [9] it is shown that if $\operatorname{gcd}\left(\frac{1}{2}\left(q^{n}+1\right), n e\right)=1$, then with the exception of Hering's plane of order 27 any non-Desarguesian flagtransitive affine plane of order $q^{n}$ whose kernel contains $\mathrm{GF}(q)$ must admit a (cyclic) Singer subgroup action which is either regular on the line at infinity or has two equal-sized orbits on the line at infinity. In the latter case these two orbits are joined by some other element in the translation complement, but there is no cyclic regular action on the line at infinity. This result holds in all known odd order nonDesarguesian flag-transitive affine planes, except Hering's, whether or not the above gcd condition is satisfied. Flag-transitive affine planes of the first type will be called $\mathscr{C}$-planes, and those of the second type will be called $\mathscr{H}$-planes.

By a celebrated result of Wagner [16] any finite flag-transitive affine plane will necessarily be a translation plane, and thus can be constructed from some $(n-1)$ spread of PG $(2 n-1, q)$. We call the spread of type $\mathscr{C}$ or $\mathscr{H}$ accordingly as the associated plane is of type $\mathscr{C}$ or $\mathscr{H}$, respectively, as defined above. When $n=2$, it can be shown that there are no type $\mathscr{C} 1$-spreads of $\mathrm{PG}(3, q)$, and the type $\mathscr{H} 1$-spreads have been completely classified (see [3]). When $n=3$, type $\mathscr{C}$ and type $\mathscr{H} 2$-spreads of $\mathrm{PG}(5, q)$ are known to exist, and the type $\mathscr{C}$ 2-spreads of $\mathrm{PG}(5, q)$ have been classified (see [1], [2], [4]). The purpose of this paper is to classify the type $\mathscr{H} 2$-spreads

* Research partly supported by NSA grant MDA 904-00-1-0029. 
of $\operatorname{PG}(5, q)$, and hence classify all odd order three-dimensional flag-transitive affine planes, subject to the above gcd condition.

It should be remarked that in any dimension type $\mathscr{H}$ flag-transitive affine planes cannot exist for even $q$, since $q^{n}+1$ must be even for such planes to exist. Moreover, type $\mathscr{C}$ flag-transitive affine planes are only known to exist for odd $n$, independent of $q$, although their existence for even $n>2$ remains an open question.

\section{Singer orbits}

Let $\Sigma=\mathrm{PG}(2 n-1, q)$ denote the $(2 n-1)$-dimensional projective space over the finite field $\operatorname{GF}(q)$, where $q$ is an odd prime power and $n \geqslant 3$ is an odd integer. We model $\Sigma$ using the finite field $K=\mathrm{GF}\left(q^{2 n}\right)$. Let $\beta$ be a primitive element for $K$. Hence, the field elements

$$
1, \beta, \beta^{2}, \ldots, \beta^{\left(q^{2 n}-1\right) /(q-1)-1}
$$

represent the distinct projective points of $\Sigma$. The collineation $\theta$ induced by multiplication by $\beta$ is a Singer cycle of $\Sigma$, and we consider the fibration $\mathscr{F}$ whose elements are the point orbits under the cyclic collineation group $G$ generated by $\theta^{N}$, where $N=\frac{q^{n}-1}{q-1}$. Thus $\mathscr{F}$ has $N$ members, each of size $M=q^{n}+1$. More precisely, $\mathscr{F}=\left\{\Omega_{i}: i=0,1,2, \ldots, N-1\right\}$, where $\Omega_{i}=\beta^{i} \Omega_{0}$ and $\Omega_{0}=\left\{\beta^{s N}: s=0,1,2, \ldots\right.$, $M-1\}$.

We start by examining how a line of $\Sigma$ can meet the fibration $\mathscr{F}$. The arguments are very similar to those found in [8].

Lemma 1. Let $A, B$, and $C$ be three distinct collinear points of $\Omega_{t}$ for some $t$. Then the line containing $A, B$, and $C$ is completely contained in $\Omega_{t}$. Moreover, this is the only line through $A$ which is contained in $\Omega_{t}$.

Proof. We may assume without loss of generality that the three collinear points are in $\Omega_{0}$, and that $A$ is represented by the field element $\beta^{0}=1$. Then $B$ and $C$ are represented by field elements of the form $\beta^{i N}$ and $\beta^{j N}$, respectively, where $1 \leqslant i<$ $j \leqslant M-1$. The three points are collinear if and only if $1+a \beta^{i N}=b \beta^{j N}$, for some $a, b \in \mathrm{GF}(q)^{*}$.

Now $\left(1+a \beta^{i N}\right)^{q^{n}+1}=\left(b \beta^{j N}\right)^{q^{n}+1}$, where $\left(b \beta^{j N}\right)^{q^{n}+1}=b^{2} \beta^{j N M} \in \mathrm{GF}(q)$, and

$$
\begin{aligned}
\left(1+a \beta^{i N}\right)^{q^{n}+1} & =\left(1+a \beta^{i N}\right)\left(1+a \beta^{i N}\right)^{q^{n}} \\
& =1+a \beta^{i N}+a \beta^{i N q^{n}}+a^{2} \beta^{i N M} .
\end{aligned}
$$

Since $a^{2} \beta^{i N M} \in \mathrm{GF}(q)$, we have $\beta^{i N}+\beta^{i N q^{n}} \in \mathrm{GF}(q)$.

Hence $f(x)=\left(x-\beta^{i N}\right)\left(x-\beta^{i N q^{n}}\right)$ is a quadratic polynomial over $\operatorname{GF}(q)$ having $\beta^{i N}$ as a root. Thus $\beta^{i N}$ is in $\operatorname{GF}\left(q^{2}\right)$, which further implies that $\beta^{j N} \in \mathrm{GF}\left(q^{2}\right)$ by the above dependency relation. Hence the three given collinear points lie on the line represented by the subfield $\mathrm{GF}\left(q^{2}\right)$. 
To show that this line is completely contained in $\Omega_{0}$, we observe that $(q+1) \mid M$ since $n$ is odd. Hence, as $\beta^{N M /(q+1)}$ is a primitive element of $\operatorname{GF}\left(q^{2}\right)$, all points on the line represented by $\operatorname{GF}\left(q^{2}\right)$ lie in $\Omega_{0}$ by definition of $\Omega_{0}$. The fact that there is only one line through $A$ completely in $\Omega_{0}$ follows from the uniqueness of the subfield $\operatorname{GF}\left(q^{2}\right)$.

It should be noted that the "ruling lines" completely contained in the $G$-orbits $\Omega_{t}$ form a geometric 1 -spread of $\operatorname{PG}(2 n-1, q)$ whenever $n$ is odd, and there are no such ruling lines when $n$ is even.

We now define $\ell_{s}$ to be the line joining the points $\beta^{0}$ and $\beta^{s N}$ of $\Omega_{0}$. From the proof of the previous lemma, $\ell_{s}$ is completely contained in $\Omega_{0}$ if and only if $s$ is a multiple of $\frac{M}{q+1}$.

Theorem 2. Suppose sis not a multiple of $\frac{M}{q+1}$, so that $l_{s}$ is not contained in $\Omega_{0}$.

1. If $s$ is odd, then $l_{s}$ is secant to exactly $\frac{q+1}{2}$ elements of $\mathscr{F}$ and is disjoint from all other members of $\mathscr{F}$.

2. If $s$ is even, then $l_{s}$ is tangent to exactly 2 elements of $\mathscr{F}$ and is secant to $\frac{q-1}{2}$ elements of $\mathscr{F}$. Moreover, the points of tangency correspond to $\beta^{s N} \pm \beta^{s N M / 2}$.

Proof. Since $s$ is not a multiple of $\frac{M}{q+1}$, we know from Lemma 1 that $l_{s}$ meets $\Omega_{0}$ in only two points. The argument now follows exactly as in the proof of Theorem 4 in $[8]$.

Hence, from Theorem 2, we know that there are four possible ways in which a line can meet $\mathscr{F}$, as listed below. We have shown that lines of the first three types exist. Direct counting shows that lines of the fourth type also must exist for any odd integer $n \geqslant 3$.

Ruling lines-lines completely contained in an element of $\mathscr{F}$. In particular, there are $q^{4}+q^{2}+1$ such lines when $n=3$.

Secant Lines-lines which meet exactly $\frac{q+1}{2}$ elements of $\mathscr{F}$ in two distinct points and are disjoint from the remaining $N-\frac{q+1}{2}$ elements of $\mathscr{F}$. There are $\frac{1}{2} q(q-1)$. $\left(q^{2}+q+1\right)\left(q^{3}+1\right)$ such lines when $n=3$.

Tangent Type Lines-lines which meet exactly 2 elements of $\mathscr{F}$ in one point, meet exactly $\frac{q-1}{2}$ elements of $\mathscr{F}$ in two distinct points, and are disjoint from the remaining $N-\frac{q+3}{2}$ elements of $\mathscr{F}$. There are $\frac{1}{2} q(q+1)\left(q^{2}+q+1\right)\left(q^{3}+1\right)$ such lines when $n=3$.

Purely Tangent Lines-lines which meet exactly $q+1$ elements of $\mathscr{F}$ in a unique point and are disjoint from the remaining $N-(q+1)$ elements of $\mathscr{F}$. There are $q\left(q^{4}+q^{2}+1\right)\left(q^{3}+1\right)$ such lines when $n=3$.

\section{The fibration in $\operatorname{PG}(5, q)$}

We now apply the theory from the last section to the special case when $n=3$; that is, $\Sigma=\operatorname{PG}(5, q)$. Hence our fibration $\mathscr{F}$ has exactly $N=q^{2}+q+1$ elements, each 
containing $M=q^{3}+1$ distinct points. As discussed in [7], there is a special collection of $q^{2}+q+1$ elliptic quadrics of $\Sigma$, each of which is partitioned into $q+1$ distinct elements of $\mathscr{F}$. Moreover, each member $\Omega_{i}$ of $\mathscr{F}$ is contained in $q+1$ of these quadrics, any two of which intersect precisely in $\Omega_{i}$. In short, if we call the elements of $\mathscr{F}$ "points" and the associated quadrics "lines", the induced incidence structure is a projective plane of order $q$.

We now define $H$ to be the unique Singer subgroup of index two in $G$. That is, $H$ is generated by the collineation $\theta^{2 N}$. This is the subgroup associated with type $\mathscr{H}$ flagtransitive affine planes, as discussed in Section 1. To that end, let $\pi$ be a projective plane of $\Sigma$ which meets each $H$-orbit in at most one point. Then $\pi$ meets each of the $G$-orbits (i.e. elements of $\mathscr{F}$ ) in at most two points, as each $G$-orbit is a union of two $H$-orbits.

Lemma 3. The plane $\pi$ contains no tangent type lines.

Proof. Suppose the plane $\pi$ contained a tangent type line, which we may assume is $l_{s}$ for some $s$. From Theorem 2 we know that $s$ is even. But then the field elements $\beta^{0}$ and $\beta^{s N}$ induce distinct points which are in the same orbit under the group $H$, contradicting the definition of $\pi$.

We have thus determined that the plane $\pi$ can only contain secant lines and purely tangent lines, as defined at the end of the previous section.

Theorem 4. The plane $\pi$ is tangent to each of the $q^{2}+q+1$ elements of $\mathscr{F}$.

Proof. For contradiction, suppose that $\pi$ meets an element of $\mathscr{F}$, say $\Omega_{j}$, in two points $P$ and $Q$. Then, by Lemma 3 , the line $l$ containing $P$ and $Q$ is a secant line. Now, if $\pi$ contained another secant line, say $m$, then $l$ and $m$ must meet in a common point, say $R$, contained in some element of $\mathscr{F}$, say $\Omega_{k}$. It follows that $\pi$ meets $\Omega_{k}$ in 3 distinct points, a contradiction. Hence, $\pi$ contains only one secant line, namely $l$, and there are exactly $\frac{q+1}{2}$ elements of $\mathscr{F}$ meeting $\pi$ in exactly two points.

Recall that $\Omega_{j}$ may be expressed as the intersection of two elliptic quadrics of $\Sigma$, as discussed above, and any two such quadrics determine an algebraic pencil of quadrics in $\Sigma$. Moreover, the plane $\pi$ intersects this pencil in a planar pencil $\mathscr{P}$ of quadrics. From the classification of planar pencils of quadrics [11], we know that one of the quadrics in $\mathscr{P}$ is a pair of distinct lines. From the discussion at the beginning of this section, this pair of lines covering $2 q+1$ points meets exactly $q+1$ elements of $\mathscr{F}$. Hence the plane $\pi$ must meet at least $q$ elements of $\mathscr{F}$ in two distinct points, contradicting our computation in the previous paragraph. That is, $\pi$ cannot meet any element of $\mathscr{F}$ in two distinct points, and the result follows.

\section{Type $\mathscr{H}$ spreads in PG(5,q)}

Let $\mathscr{S}$ be a type $\mathscr{H} 2$-spread in $\Sigma=\mathrm{PG}(5, q)$, where $q$ is an odd prime power. In particular, $\mathscr{S}$ consists of $q^{3}+1$ mutually disjoint planes, necessarily partitioning the 
points of $\Sigma$. Since Singer subgroups are unique up to conjugation, we may use the Singer subgroups $H$ and $G$ of the previous section. By definition of type $\mathscr{H}$ spreads and the fact that the only short orbit on planes of the full Singer group is a regular 2spread (see [10], for instance), we know that $\mathscr{S}=\pi_{1}^{H} \cup \pi_{2}^{H}$, where $\pi_{1}$ and $\pi_{2}$ are two skew planes of $\Sigma$. Moreover, $\mathscr{S}$ is not a single plane orbit under $G$. Since the planes in $\mathscr{S}$ are mutually disjoint, $\pi_{1}$ (and similarly, $\pi_{2}$ ) meets each point orbit under $H$ in at most one point. This implies by Theorem 4 that $\pi_{1}$ (and similarly, $\pi_{2}$ ) necessarily meets each of the $q^{2}+q+1$ point orbits under $G$ in exactly one point, and thus is a "purely tangent plane" with respect to the fibration $\mathscr{F}$ of $G$-orbits.

Since $\pi_{1}$ is a purely tangent plane with respect to $\mathscr{F}$, the classification of type $\mathscr{C}$ spreads in [2] and [4] implies that $\pi_{1}$ is projectively equivalent (in fact, by a Singer shift) to a plane represented by a GF $(q)$-subspace of the form $S_{b}=\left\{z+b z^{q}\right.$ : $\left.z \in \operatorname{GF}\left(q^{3}\right)\right\}$, for some $b \in K=\operatorname{GF}\left(q^{6}\right)$ such that $b^{q^{3}}=-b$. Thus the fundamental issue is determining how many compatible half-spreads $\pi_{2}^{H}$ exist for a given halfspread $\pi_{1}^{H}$.

Theorem 5. Let $\pi^{H}$ be some cyclic half-spread in $\mathrm{PG}(5, q)$, where $q$ is an odd prime power. Then, unless $\pi^{H}$ is contained in a regular spread, there is precisely one way of completing $\pi^{H}$ to a type $\mathscr{H}$ spread and precisely one way of completing $\pi^{H}$ to a type $\mathscr{C}$ spread. If $\pi^{H}$ is contained in a regular spread, then there is only one way of completing $\pi^{H}$ to either a type $\mathscr{H}$ or type $\mathscr{C}$ spread, and that completion is the unique regular spread containing $\pi^{H}$.

Proof. As shown above, $\pi$ is necessarily a purely tangent plane with respect to the fibration $\mathscr{F}$ of $G$-orbits, and thus $\pi^{G}$ is a type $\mathscr{C}$ spread completing the half-spread $\pi^{H}$. Moreover, we know the plane $\pi$ is a Singer shift of one represented by some subspace $S_{b}$, where $b^{q^{3}}=-b$. Thus from the work in [12] and [14] we know there is at least one way of completing $\pi^{H}$ to a spread of type $\mathscr{H}$, as long as $b \neq 0$.

Suppose now that $\pi_{1}^{H}$ and $\pi_{2}^{H}$ are two completions of the half-spread $\pi^{H}$ to a spread that is either of type $\mathscr{H}$ or of type $\mathscr{C}$. Then by the same argument as that given above, we know that $\pi_{1}$ and $\pi_{2}$ must be purely tangent planes with respect to the fibration $\mathscr{F}$. By translating appropriately and shifting our point of view, we may assume both $\pi_{1}$ and $\pi_{2}$ pass through the point represented by $\beta^{0}$, and hence each is represented by a subspace of the form $\frac{1}{1+b} S_{b}=\left\{\frac{x+b x^{q}}{1+b}: x \in \operatorname{GF}\left(q^{3}\right)\right\}$, for some $b \in K$ with $b^{q^{3}}=-b$. Note that the collineation induced by multiplication by $(1+b)^{-1}$ is a power of the Singer cycle, and the shifted plane $(1+b)^{-1} S_{b}$ is a purely tangent plane passing through $\beta^{0}$. With a bit of computation, which will soon become apparent, one can show that distinct values of $b$ determine distinct planes $(1+b)^{-1} S_{b}$, and hence we get as a byproduct that there are exactly $q^{3}$ purely tangent planes through a given point, such as $\beta^{0}$, for any odd prime power $q$.

Let $B=\left\{b: b \in K, b^{q^{3}}=-b\right\}$. Now the existence of the above two completions of $\pi^{H}$ implies the existence of $b_{1}, b_{2} \in B$ such that $\left(1+b_{1}\right)^{-1} S_{b_{1}}$ and $\left(1+b_{2}\right)^{-1} S_{b_{2}}$ represent planes, namely $\pi_{1}$ and $\pi_{2}$, that meet the same point orbits under $H$. Since $H=\left\langle\theta^{2\left(q^{2}+q+1\right)}\right\rangle$, this means that for each $x \in \operatorname{GF}\left(q^{3}\right)$, there exists some $y \in \operatorname{GF}\left(q^{3}\right)$ such that 


$$
\left(\frac{x+b_{1} x^{q}}{1+b_{1}}\right)^{q^{3}+1}=\gamma\left(\frac{y+b_{2} y^{q}}{1+b_{2}}\right)^{q^{3}+1}
$$

for some nonzero square $\gamma$ in $\operatorname{GF}(q)$. Moreover, we may chose an appropriate nonzero $\operatorname{GF}(q)$-multiple of $y$ so that $\gamma=1$. Thus, for every $x \in \operatorname{GF}\left(q^{3}\right)$, there exists some $y$ in $\mathrm{GF}\left(q^{3}\right)$ such that

$$
\frac{x^{2}-b_{1}^{2} x^{2 q}}{1-b_{1}^{2}}=\frac{y^{2}-b_{2}^{2} y^{2 q}}{1-b_{2}^{2}}
$$

where $b_{1}$ and $b_{2}$ are fixed elements of $B$.

For each $b \in B$, consider the mapping $T_{b}: \mathrm{GF}\left(q^{3}\right) \rightarrow \mathrm{GF}\left(q^{3}\right)$ via $T_{b}: z \rightarrow \frac{z-b^{2} z^{4}}{1-b^{2}}$. Since $b^{2}$ is either 0 or a nonsquare in $\operatorname{GF}\left(q^{3}\right), T_{b}$ is a nonsingular linear transformation of the vector space $\mathrm{GF}\left(q^{3}\right)$ over $\mathrm{GF}(q)$, and hence $T_{b}$ is a permutation polynomial of the field $\mathrm{GF}\left(q^{3}\right)$. Moreover, each $T_{b}$ fixes each of the elements 0 and 1 . Hence the composition $T_{b_{1}} \circ T_{b_{2}}^{-1}$ is a $\mathrm{GF}(q)$-linear permutation polynomial with the previously mentioned properties that also maps squares to squares by Equation (1), and thus preserves quadratic character. By the main theorem in Carlitz [6] $T_{b_{1}} \circ T_{b_{2}}^{-1}$ is necessarily one of the following three maps: $z \rightarrow z, z \rightarrow z^{q}$, or $z \rightarrow z^{q^{2}}$. In particular, this implies that $x^{2}=y^{2}, x^{2}=y^{2 q}$, or $x^{2}=y^{2 q^{2}}$ in Equation (1) above. Now take an appropriate linear combination to solve for $x^{2}$, say $\frac{1}{b^{2(q+1)}}$ times Equation (1) plus $\frac{b_{1}^{2}}{\left(1-b_{1}^{2}\right)^{q^{2}+1}}$ times the $q$-th power of (1) plus $\frac{b_{1}^{2(q+1)}}{\left(1-b_{1}^{2}\right)^{q+1}}$ times the $q^{2}$-th power of (1), and simplify. One obtains the equation

$$
\begin{aligned}
\frac{1-b_{1}^{2\left(q^{2}+q+1\right)}}{\left(1-b_{1}^{2}\right)^{q^{2}+q+1}} x^{2}= & \left(\frac{1-b_{1}^{2\left(q^{2}+q+1\right)}}{\left(1-b_{1}^{2 q}\right)\left(1-b_{1}^{2 q^{2}}\right)\left(1-b_{2}^{2}\right)}+b_{1}^{2(q+1)} N\right) y^{2} \\
& +N^{q} y^{2 q}+b_{1}^{2} N^{q^{2}} y^{2 q^{2}}
\end{aligned}
$$

where $N=\frac{b_{1}^{2 q^{2}}}{\left(1-b_{2}^{2}\right)\left(1-b_{1}^{2 q}\right)\left(1-b_{1}^{24^{2}}\right)}-\frac{b_{2}^{2 q^{2}}}{\left(1-b_{2}^{24^{2}}\right)\left(1-b_{1}^{2 q}\right)\left(1-b_{1}^{2}\right)}$. By the Carlitz result above, we must have $N=0$ and thus $\frac{x^{2}}{1-b_{1}^{2}}=\frac{y^{2}}{1-b_{2}^{2}}$. Since $T_{b_{1}} \circ T_{b_{2}}^{-1}$ fixes the element 1 , we must have $x^{2}=1$ if and only if $y^{2}=1$, and thus $b_{1}= \pm b_{2}$.

This implies that there are precisely two ways of completing the half-spread $\pi^{H}$ to a full spread of type $\mathscr{C}$ or type $\mathscr{H}$, provided $b_{1} \neq 0$. Namely, if we think of $\pi_{1}$ as $\pi^{\theta^{2^{2}+q+1}}$, then choosing $b_{2}=b_{1}$ will yield the type $\mathscr{C}$ completion $\pi^{H} \cup \pi_{1}^{H}$, while choosing $b_{2}=-b_{1}$ will yield the type $\mathscr{H}$ completion $\pi^{H} \cup \pi_{2}^{H}$ as described in [12] and [14]. If $b_{1}=0$, then necessarily $b_{2}=0$ and the only possible completion is the regular spread (see [12]). This completes the proof.

Corollary 6. All odd order three-dimensional flag-transitive affine planes of type $\mathscr{H}$ or $\mathscr{C}$ are known. If the order is $q^{3}$, so that the kernel is $\mathrm{GF}(q)$, the number of isomorphism 
classes of each type is at least $(q-1) / 2 e$, where $q=p^{e}$ and $p$ is an odd prime. Furthermore, if $q$ is a prime, then the number of isomorphism classes of each type is exactly $(q-1) / 2$. Finally, if $\operatorname{gcd}\left(\frac{1}{2}\left(q^{3}+1\right), 3 e\right)=1$, then with the exception of Hering's plane of order 27 every non-Desarguesian flag-transitive affine plane of order $q^{3}$ with kernel $\mathrm{GF}(q)$ is necessarily of type $\mathscr{C}$ or type $\mathscr{H}$, and hence is known.

Proof. The classification of type $\mathscr{C}$ flag-transitive affine planes of odd order $q^{3}$ follows from the work in [1], [2], and [4] as discussed above, while the classification in the type $\mathscr{H}$ case follows from the previous theorem. The isomorphism counts are given in [12] and [14] (see also [2]). The final statement is a special case of Lemma 1 in [9].

Thus, modulo the gcd condition stated in the above corollary, the only possible flag-transitive affine planes of odd order $q^{3}$ are those constructed by Kantor and Suetake in [12] and [14], together with Hering's plane of order 27. When $q=5$, the gcd condition is not satisfied, and yet exhaustive searching in [15] shows that there are no other flag-transitive affine planes of order 125. Perhaps the gcd condition is superfluous.

\section{Concluding remarks}

The key to classifying the type $\mathscr{H}$ flag-transitive affine planes of odd order $q^{3}$ is showing that if $\pi^{H}$ is a half-spread, then $\pi$ must be tangent to each of the $G$-orbits in the fibration $\mathscr{F}$. Once this is known, a short proof of the classification in both the type $\mathscr{C}$ and type $\mathscr{H}$ cases could be given if one could prove directly that there are precisely $q^{3}$ purely tangent planes with respect to $\mathscr{F}$ that pass through a given point, say $\beta^{0}$. This would eliminate the reduction to Baer subplane partitions in [1], the very involved use of linearized polynomials in [2], and the messy cyclotomic lemma in [4]. Unfortunately, we have so far been unable to do this counting directly.

When $q$ is even, computer searches using MAGMA [5] show that for small values of $q$ there are precisely $\left(q^{2}+1\right)(q+1)$ purely tangent planes with respect to the fibration $\mathscr{F}$ that pass through a given point, say $\beta^{0}$. Again, if one could directly prove that this result always holds, one would essentially have a classification of the flagtransitive affine planes of type $\mathscr{C}$ with even order $q^{3}$ (there are none of type $\mathscr{H}$ ). Namely, based on the known construction (succinctly described in [13]), one can show that there are at least $\left(q^{2}+1\right)(q+1)$ purely tangent planes passing through $\beta^{0}$ when $q$ is even, and hence all would be known.

In higher dimensions the problem is trickier. Consider a type $\mathscr{H}$ flag-transitive affine plane of order $q^{n}$, where $q$ is necessarily odd, and let $\pi^{H}$ be one of the associated cyclic half-spreads of $\operatorname{PG}(2 n-1, q)$. If $n$ is odd, in all known examples $\pi$ meets each $G$-orbit in exactly one point and thus is "purely tangent" with respect to the fibration $\mathscr{F}$. Hence there is a companion type $\mathscr{C}$ flag-transitive affine plane with associated spread $\pi^{G}$. When $n=3$, we used (in Section 3) the classification of pencils of 
quadrics in $\mathrm{PG}(2, q)$ to show that such an intersection pattern must happen. Proving that this pattern must hold for all odd $n>3$ would be a big step toward a higherdimensional classification. Perhaps this could be done without the complete classification of pencils of quadrics in higher dimensions, which is as yet unknown.

When $n$ is even, all known examples of type $\mathscr{H}$ flag-transitive affine planes with associated spread $\pi_{1}^{H} \cup \pi_{2}^{H}$ are such that $\pi_{1}$ meets half the $G$-orbits of $\mathscr{F}$ in two points each (from different $H$-orbits), while $\pi_{2}$ meets the other half of the $G$-orbits in two points each. When $n=2, \pi_{1}$ and $\pi_{2}$ are lines and it is straightforward to show that this must be the case (see [8]). For even $n \geqslant 4$, it is unclear at present why (or if) this pattern must hold. In particular, it would be nice to know (for $q$ odd or $q$ even) if there exists a purely tangent $(n-1)$-space with respect to $\mathscr{F}$ for even $n \geqslant 4$, other than the example where the resulting cyclic spread is regular and hence the plane is Desarguesian.

\section{References}

[1] R. D. Baker, J. M. Dover, G. L. Ebert, K. L. Wantz, Baer subgeometry partitions. J. Geom. 67 (2000), 23-34. MR 2001g:51010 Zbl 0974.51005

[2] R. D. Baker, J. M. Dover, G. L. Ebert, K. L. Wantz, Perfect Baer subplane partitions and three-dimensional flag-transitive planes. Des. Codes Cryptogr. 21 (2000), 19-39. MR 2002a:51010 Zbl 0970.51006

[3] R. D. Baker, G. L. Ebert, Two-dimensional flag-transitive planes revisited. Geom. Dedicata 63 (1996), 1-15. MR 97j:51016 Zbl 0868.51005

[4] R. D. Baker, G. L. Ebert, K. H. Leung, Q. Xiang, A trace conjecture and flag-transitive affine planes. J. Combin. Theory Ser. A 95 (2001), 158-168. MR 2002c:11166 Zbl 0985.51007

[5] J. Cannon, C. Playoust, An Introduction to MAGMA. Univ. of Sydney, Australia, 1993.

[6] L. Carlitz, A theorem on permutations in a finite field. Proc. Amer. Math. Soc. 11 (1960), 456-459. MR 22 \#8005 Zbl 0095.03003

[7] A. Cossidente, L. Storme, Caps on elliptic quadrics. Finite Fields Appl. 1 (1995), 412-420. MR 96j:51014 Zbl 0845.51008

[8] G. L. Ebert, Partitioning projective geometries into caps. Canad. J. Math. 37 (1985), 1163-1175. MR 87h:51024 Zbl 0571.51002

[9] G. L. Ebert, Partitioning problems and flag-transitive planes. Rend. Circ. Mat. Palermo (2) Suppl. no. 53 (1998), 27-44. MR 99h:51007 Zbl 0926.51012

[10] G. L. Ebert, K. Metsch, T. Szönyi, Caps embedded in Grassmannians. Geom. Dedicata 70 (1998), 181-196. MR 99c:51016 Zbl 0911.51013

[11] J. W. P. Hirschfeld, Projective geometries over finite fields. Oxford Univ. Press 1998. MR 99b:51006 Zbl 0899.51002

[12] W. M. Kantor, Two families of flag-transitive affine planes. Geom. Dedicata 41 (1992), 191-200. MR 93d:51019 Zbl 0748.51004

[13] W. M. Kantor, 2-transitive and flag-transitive designs. In: Coding theory, design theory, group theory (Burlington, VT, 1990), 13-30, Wiley, New York 1993. MR 94e:51018

[14] W. M. Kantor, C. Suetake, A note on some flag-transitive affine planes. J. Combin. Theory Ser. A 65 (1994), 307-310. MR 95e:51012 Zbl 0801.51005

[15] A. R. Prince, A complete classification of the flag-transitive affine planes of order 125. J. Combin. Des. 5 (1997), 147-153. MR 97j:51012 Zbl 0934.51007 
[16] A. Wagner, On finite affine line transitive planes. Math. Z. 87 (1965), 1-11. MR 30 \#2391 Zbl 0131.36804

Received 20 September, 2002

R. D. Baker, Department of Mathematics, West Virginia State College Institute, WV $25112-$ 1000, USA

Email: baker@aeross.wvsc.edu

C. Culbert, G. L. Ebert, Department of Mathematical Sciences, University of Delaware, Newark, DE 19716-2553, USA

Email: ebert@math.udel.edu

K. E. Mellinger, Department of Mathematics, Statistics and Computer Science, University of Illinois at Chicago, Chicago, IL 60607-7045, USA

Email: kmelling@math.uic.edu 